\title{
Interaction of von Karman vortices and intersecting main streams in staggered tube bundles
}

\author{
S. Umeda, W.-J. Yang
}

\begin{abstract}
Flow visualization, heat transfer and pressure drop characteristics in flow through staggered tube bundles have been regarded as classical, with results well-documented. However, the mechanism of producing such results has been left untouched. Applications of staggered tube bundles are abundant in industry, for example as heat exchange devices like the shell-and-tube type and fuel bundles in nuclear reactor cores. An experimental study is recorded in the present paper which investigates the interaction of von Karman vortices and intersecting main streams in staggered tube bundles. Flow visualization by means of the particle tracing method, laser Doppler velocimetry $(L D V)$ and pressure drop measurements using a piezometer are conducted. A modified Reynolds number appropriate to flow through a staggered tube arrangement is defined together with a pressure drop coefficient. Auto-correlation and power spectrum analyses of signals obtained from $L D V$ measurements yield an optimum spectrum frequency which is correlated against the Reynolds number. It is concluded that flow characteristics in staggered tube bundles are determined by the interaction between the von Karman vortex street and X-shaped interacting main streams.
\end{abstract}

\section{Introduction}

A flow network consists of a family of mutually intersecting flow passages. It is abundant in the field of natural science and provides interesting phenomena with potential applications for heat transfer enhancement, flow control and others, for example (Umeda and Yang 1996a). Research results pertinent to heat exchangers with inline- and staggered-tube arrangements are available in the existing literature and heat transfer text books. A comparison of loss and heat transfer performance in the inline- and staggered tube bundles has revealed that flows in these units are investigated based on flow over a single tube case. However, in the staggered arrangement case, there forms a family of $\mathrm{X}$-shaped intersecting flows resulting

Received: 4 August 1997/ Accepted: 3 October 1998

S. Umeda

Department of Civil Engineering, Fukuyama University

Gakuen-cho, Fukuyama, Hiroshima, 729-0292 Japan

W.-J. Yang

Department of Mechanical Engineering and Applied Mechanics University of Michigan, Ann Arbor, Mi 48109, USA

Correspondence to: W.-J. Yang from a repetition of converging-diverging flows (Umeda and Yang 1996b). This situation is grossly different from flow inside the inline-arrangement case. Flow visualization in tube bundles were conducted by Werle (1972) and Rabbo and Weaver (1986). Chen (1987) summarized the phenomena of flow-induced vibration which occurred inside cylindrical tube bundles. Weaver and Fitzpatrick (1988) reviewed the literature pertinent to cross-flow induced vibrations in heat exchanger tube arrays. They classified and discussed the flow excitation mechanisms. However, those studies had not achieved a thoroughly categorized form of analyses on flow inside tube bundles. Recently, Moretti (1993) classified the fluid/solid interactions about flow-induced vibrations in arrays of cylinders occurring in cross flow and suggested that understanding the mutual interactions between these fluid phenomena and structural vibrations was a challenge. Nakai et al. (1996) conducted a study focusing on flow through tube bundles in order to clarify the mechanisms of generation of vibration and noise inside boiler tube bundles. However, it was short of sufficient flow measurements.

It is important to investigate the relation between flows and vortices in the formation of geometry (staggered in isosceles triangles) for the arrays of cylinders which produce the $\mathrm{X}$-shaped intersecting flows.

In the present study, a tracer injection method is applied to vizualize streamlines, and both piezometry and two-dimensional laser Doppler velocimetry $(L D V)$ are conducted for flow measurements. Results are obtained for friction loss characteristics and frequency analysis is performed to determine the power spectrum (i.e., spectra of pressure fluctuations) in staggered tube bundles. Note that emphasis is directed toward flows and vortex formation, while flow-induced tube vibrations are excluded from consideration.

\section{2}

\section{Experiment}

A schematic of the experimental apparatus used in the present study is illustrated in Fig. 1, a top view and b side view. It consisted of an upper tank, test section, lower tank and pump. The upper tank served as a reservoir, to supply water to the test section, with a water head (or height) of $\mathrm{Hu}$. A number of tubes were arranged in a staggered form inside the test section of length $L$ and width $b$. After flowing through the test section, the water was collected in a lower tank and then circulated back to the upper tank by means of a pump. An overflow control was installed in the upper tank to maintain the water level at $\mathrm{Hu}$.

Figure 2 shows four tubes of radius $r, \mathrm{~A}, \mathrm{~B}, \mathrm{C}$ and $\mathrm{D}$ placed in a staggered arrangement to form a diamond shape. The origin 


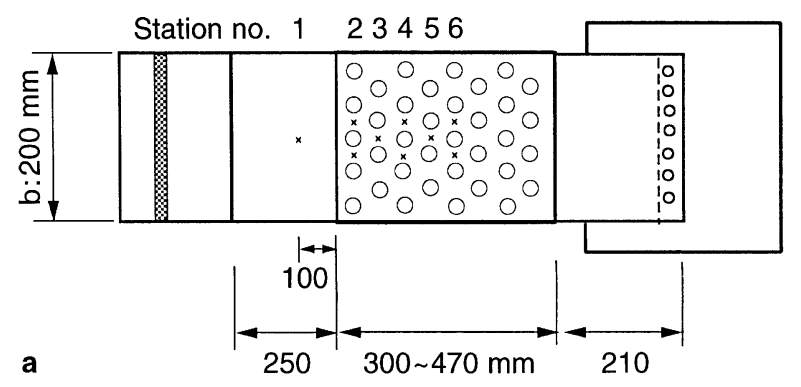

390

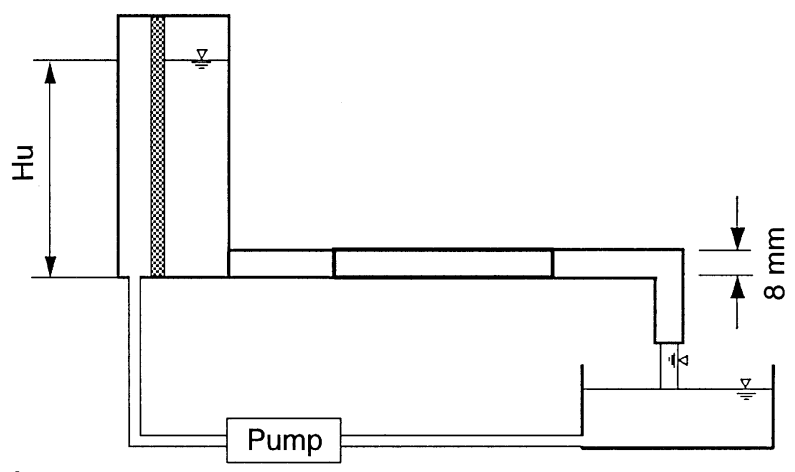

b

Fig. 1a,b. A schematic of experimental setup a Top view; b side view

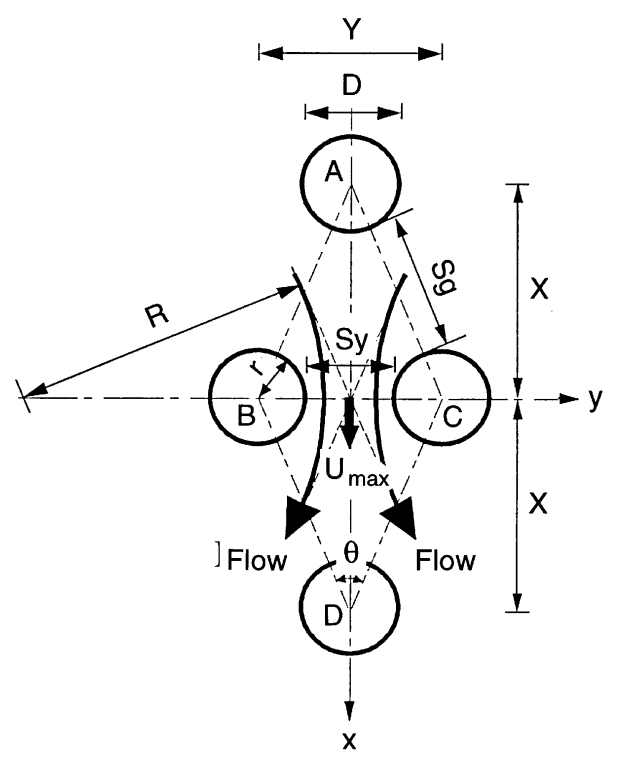

Fig. 2. A schematic diagram of flow in a tube bundle

is fixed at the center of the diamond with the $\mathrm{x}$ axis passing through the centers of tubes $A$ and $D$, and the $y$ axis passing through the centers of tubes B and C. The main stream flows in the positive $\mathrm{x}$ direction. Let the center-to-center distance of tubes A and D be $2 X$, while that of tubes B and C is Y. $S_{g}$ denotes the diagonal opening, while $S_{y}$ is the transverse opening. The latter is the difference between the transverse pitch, the distance between centers of the tubes in adjacent longitudinal rows, and the tube diameter. The experimental data for heat transfer in flow over banks of tubes is usually correlated by an equation of the form $\mathrm{Nu}=$ constant $\cdot(R e)^{m}(P r)^{n}$. Here $N u, R e$ and $\operatorname{Pr}$ denote the Nusselt, Reynolds and Prandtl numbers, respectively. Since the speed of the fluid varies along its path, it is necessary to select a reference velocity to define the Reynolds number. The reference velocity, $U_{\max }$, is based on the minimum free area available for fluid flow $A_{\min }$ per unit length of the tube length, regardless of the minimum area to occur in the transverse or diagonal opening. The Reynolds number is defined as:

$R e=\frac{U_{\max } D}{v}$

where $v$ is the kinetic viscosity. In case of an in-line tube arrangement, $A_{\min }$ is always $S_{y}$, but for a staggered arrangement, $A_{\min }$ is either $S_{y}$ or $S_{g}$ if $S_{y}$ is larger than $S_{g}$. It is known that for flow over tube bundles, the laminar-flow range is $R e<200$ and the turbulent-flow range of $R e>6000$, with the transition-flow range is between $(200<R e<6000)$. In Fig. 2, $R$ denotes the radius of curvature for flow around the tube $\mathrm{B}$ and $\mathrm{C}$ through the opening $S_{y}$. It is a fluid motion around this radius which induces the centrifugal force, as will be discussed later.

In the present study, the diameter $(D)$, number $(n)$, "staggered angle" $(\theta)$, longitudinal pitch $(X)$ and transverse pitch $(Y)$ of tubes in each test section of width $(b)$ and length $(L)$ were varied, as listed in Table 1 . Here, the "staggered angle" refers to the angle formed between two adjacent diagonal openings, as depicted in Fig. 2. For $\theta=60^{\circ}$ (i.e.; aspect ratio of 1.16), the tubes are arranged in staggered equilateral triangles, for $\theta=90^{\circ}$ (aspect ratio of 2.00), the tubes are arranged in staggered squares; and for other values of $\theta$ such as $30^{\circ}$ (aspect ratio of 0.54 ) and $45^{\circ}$ (aspect ratio of 0.81 ), the tubes are arranged in staggered in isosceles triangles. The angle $\theta$ is restricted to the range of $180^{\circ}>\theta>0^{\circ}$. The special cases of $\theta=0^{\circ}$ and $180^{\circ}$ correspond to the tube arrangements in single row and single line, respectively, or equivalently, with the aspect ratios $Y / X$ of zero and infinity, respectively.

For each tube bank, the water level in the upstream tank, $\mathrm{Hu}$, and downstream exit conditions were varied. The latter included (i) an attachment of a set of inversed-L shaped, exit-flow tubes, (ii) a change in the number of exit-flow tubes, and (iii) an open exit-flow case (in the absence of exit flow tubes). Variations in flow patterns through each tube bank with changes in the exit flow conditions were visualized by injecting high-porous, polymer particles (of an ion-exchange resin). Pressure measurement using a piezometer and velocity measurement by means of the $L D V$ method were conducted at stations marked with "cross" signs in Fig. 1. The flow velocity employed in the following analysis was either the mean velocity obtained by dividing flow rate by the flow cross-sectional area at station 1 or the velocity at the midpoint in the liquid depth measured by using $L D V$ for all other stations. The pressure and velocity at stations 2, 4 and 6 corresponded to the mean values of those measured at two locations on each side of the tubes. The length $L$ in Table 1 refers to the distance between stations 1 and 6 , while the number of tubes $n$ corresponds to that up to the fifth rows, i.e., total numbers of tubes from stations 2 to 6 . The types of tube banks listed in Table 1 include three units of $\theta=30^{\circ}$ (types 30-1, 30-2 and 30-3), one of $\theta=45^{\circ}$ (type 45-1) and two of $\theta=60^{\circ}$ (types 60-1 and 60-2), in order to determine the effects of the staggered angle $\theta$, aspect ratio $Y / X$ and tube diameter (for the same staggered arrangement). 
Table 1. Types of tube banks (Unit:mm)

\begin{tabular}{lcrrrr}
\hline Type & $30-1$ & $30-2$ & $30-3$ & $45-1$ & $60-1$ \\
\hline Diameter:D & 18 & 25 & 32 & 18 & 18 \\
Length:L & 426 & 454 & 447 & 276 & 266 \\
Width:b & 200 & 200 & 200 & 200 & 200 \\
Test Number of & 25 & 20 & 25 & 33 & 25 \\
section tube banks:n & & & & & \\
Row of tube banks:m & 5 & 5 & 5 & 8 & 8 \\
Aspect ratio: Y/X & 0.54 & 0.54 & 0.54 & 0.81 & 1.16 \\
Rate of channel & 0.925 & 0.892 & 0.775 & 0.848 & 0.880 \\
section:Ct & & & & &
\end{tabular}

\section{3}

\section{Experimental results and discussion}

For practical applications, all experiments were conducted in either the transition-flow range $6000>R e>200$ or the turbulent-flow range $R e>6000$.

\section{1}

\section{Visualization of flow patterns}

Visualization results of flow inside tube banks by means of the tracer method are illustrated in Figs. 3-11. In the type 30-1 arrangement with $\theta=30^{\circ}$, wake flow patterns behind the firstthrough fourth-row tubes varied appreciably with an increase in the Reynolds number Re. At a small value of $R e$, Fig. 3 shows a consecutive decrease in the length of the wake-flow region with the row of tubes. At a higher $R e$, an oscillation of the von Karman vortex street was seen to occur behind the first-row tubes. It propagated downstream, causing a large streamline oscillation of the main stream. As a result, a flip-flop flow was induced at the downstream end (Fig. 4). This flow oscillation phenomenon is related not only to the magnitude of $R e$ but most importantly to the fact that the diverging flow angle of the main stream confined between two adjacent longitudinal rows is smaller than the angle of its subsequent converging flow angle as seen in Fig. 3.

Figures 5 and 6 show the results of type 30-3 for the tubes of a larger diameter $D=32 \mathrm{~mm}$. For a small value of $R e, s$ $(=1450)$, the shapes of vortices behind the odd- and evennumber rows of the tubes were markedly different. With an increase in $R e, s$, the swirling of vortices behind the tubes appeared more clearly, illustrating the different shape and location of the vortices along the downstream direction. This phenomena must be related to a large difference in the converging and diverging flow angles of the main stream as well as a relatively small angle of the diverging flow. As in the case of type 30-1 tube bundle, the generation of vortices and the resulting oscillation of the main stream are the mechanisms of heat transfer enhancement in the flow over tube bundles.

In Fig. 7 for the type $45-1$ unit with $\theta=45^{\circ}$, the wake vortices behind the tubes were enclosed by the main stream up to the third-row and their size was relatively stable. However, the oscillation of the main stream, similar to that in the type 30-1 unit, began downstream from the third-row tubes.

As to the type 60-1 case with $\theta=60^{\circ}$, not much change in the vortex shape behind the tubes in the downstream direction was detected in Figs. 8 and 9. Even with an increase in $R e$, only a minor oscillation of the main stream was observed to occur locally, there was no large oscillation as previously seen in those $\theta=30^{\circ}$ units. However, the diverging flow angle appeared larger than in all other units of $\theta=30^{\circ}$ and $45^{\circ}$. Practically, no main stream oscillation was observed in Figs. 10 and 11 for the type 60-2, with almost the same size of stable vortices behind the tubes. Umeda and Yang (1995) found that in a flow through two intersecting ducts with a $60^{\circ}$ intersecting angle, two uneven inlet flow streams may result in an equalization of two downstream exit flows after their collision in the intersecting section and a subsequent formation of vortices in the downstream ducts. A similar phenomenon happens in the tube bundle staggered in equilateral triangles $\left(\theta=60^{\circ}\right)$ : the main stream restrains the vortices generated behind the tubes, producing nearly symmetrical flows around each tube and almost symmetrical pairs of vortices behind the tubes. It is thus feasible to apply tubes staggered in equilateral triangles to fluid control for equalizing velocity and flow rate.

3.2

\section{Resistance characteristic of flow passages}

It was disclosed through flow visualization in the preceding section that flow in a staggered tube arrangement is complicated

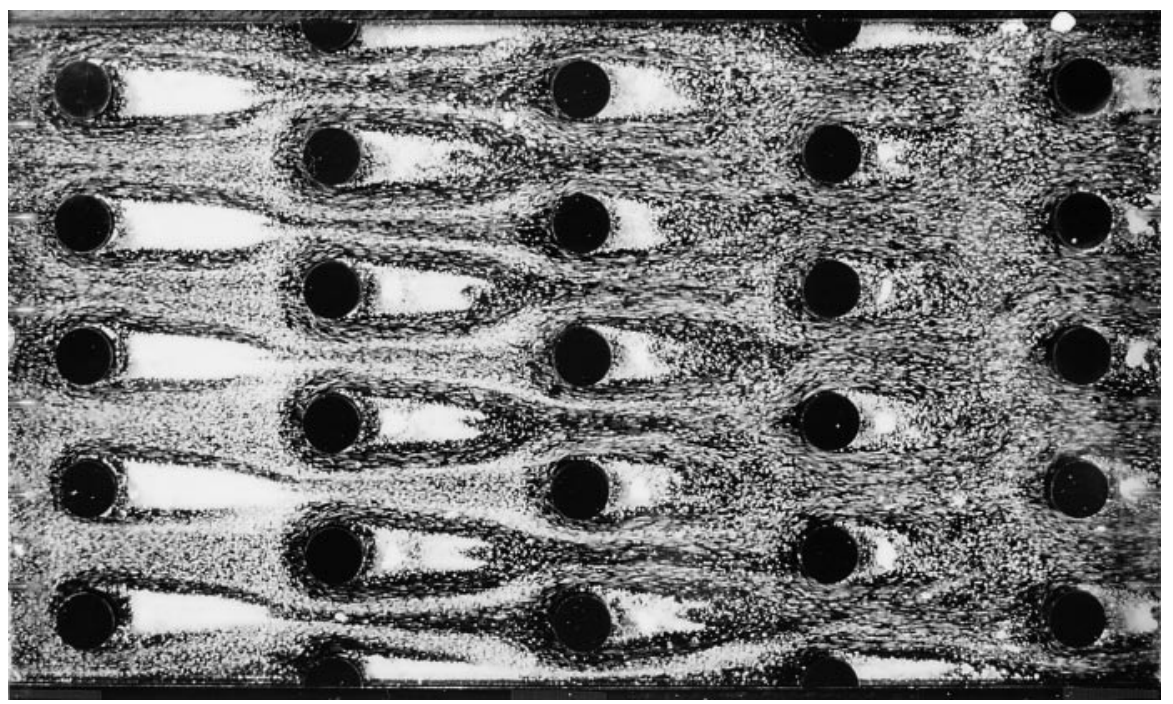

Fig. 3. Flow patterns in type $30-1$ tube bank at $R e, s=860$ 

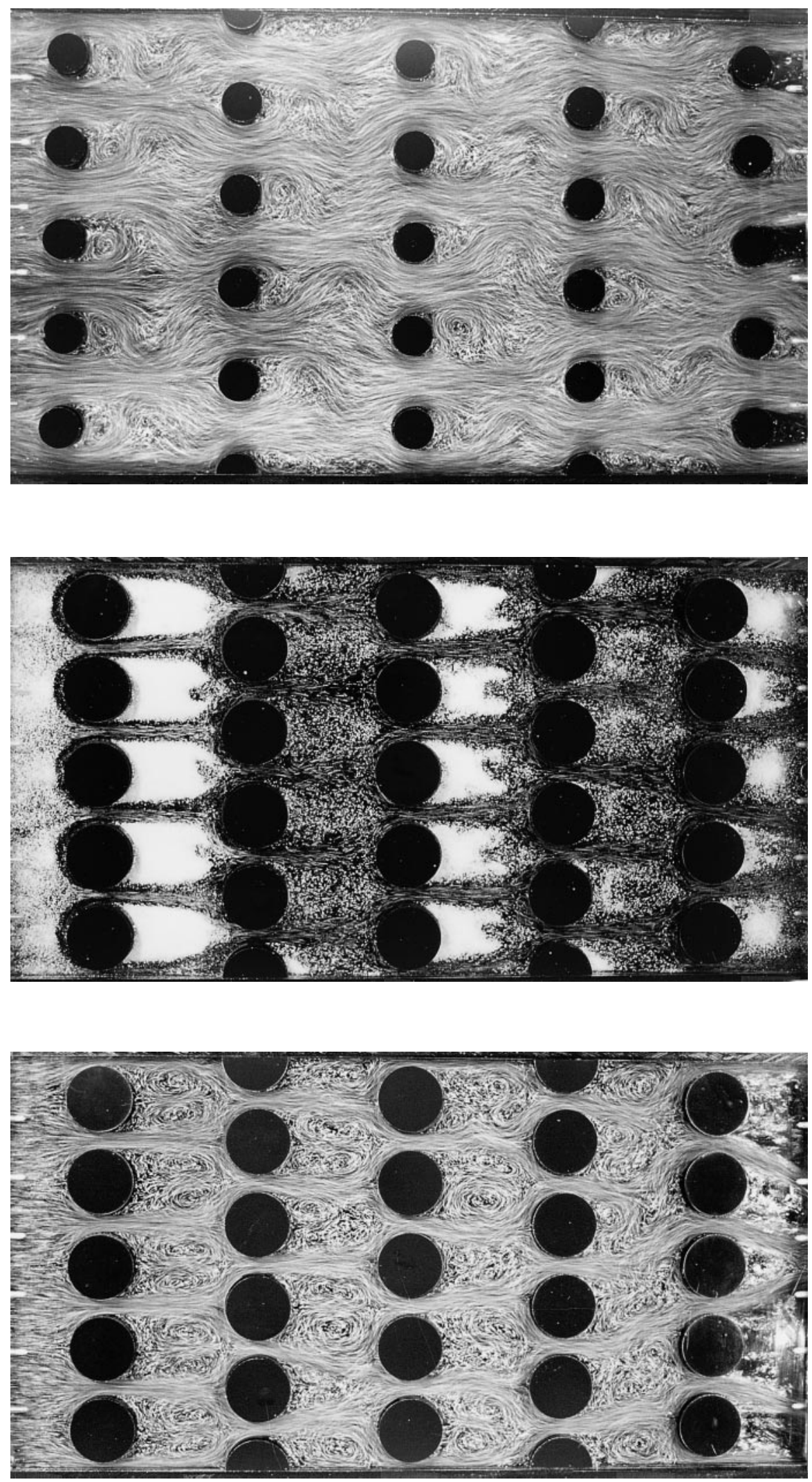

Fig. 4. Flow patterns in type $30-1$ tube bank at $\operatorname{Re}, s=16990$

Fig. 5. Flow patterns in type $30-3$ tube bank at $\operatorname{Re}, s=1450$

Fig. 6. Flow patterns in type $30-3$ tube bank at $R e, s=15750$ resulting from an interaction of the main flows through spaces between tubes and a wake flow behind each tube. These main flows meet to form an X-shaped intersecting flow in the lateral space between two neighboring tubes, B and C in Fig. 2, a phenomenon that is absent in the cases of flow over a single tube and of flow through an in-lined tube bundle. This $\mathrm{X}$-shaped intersecting flow plays two different roles: First, it can be regarded as a composite of a pair of converging and diverging flows, resembling flow in two intersecting passages (Umeda and Yang 1996b). Second, it can be regarded as a pair 


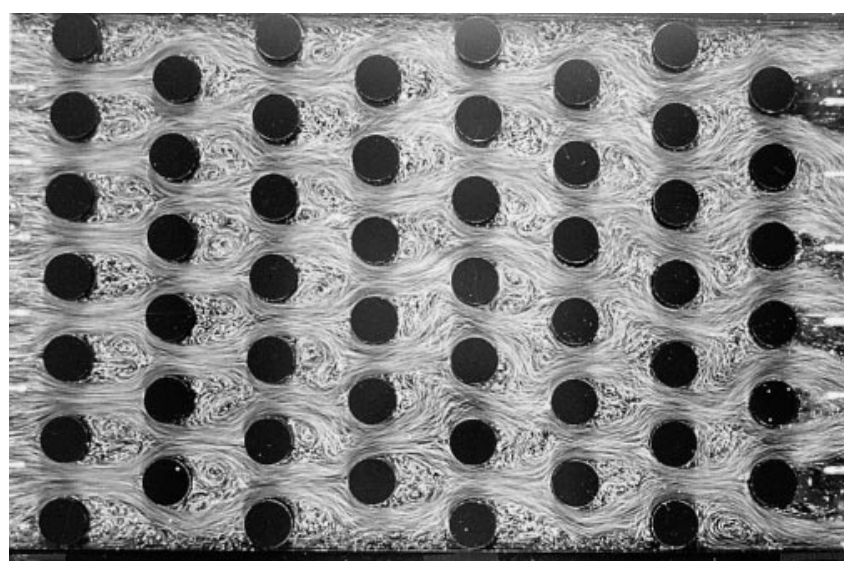

Fig. 7. Flow patterns in type $45-1$ tube bank at $R e, s=13430$

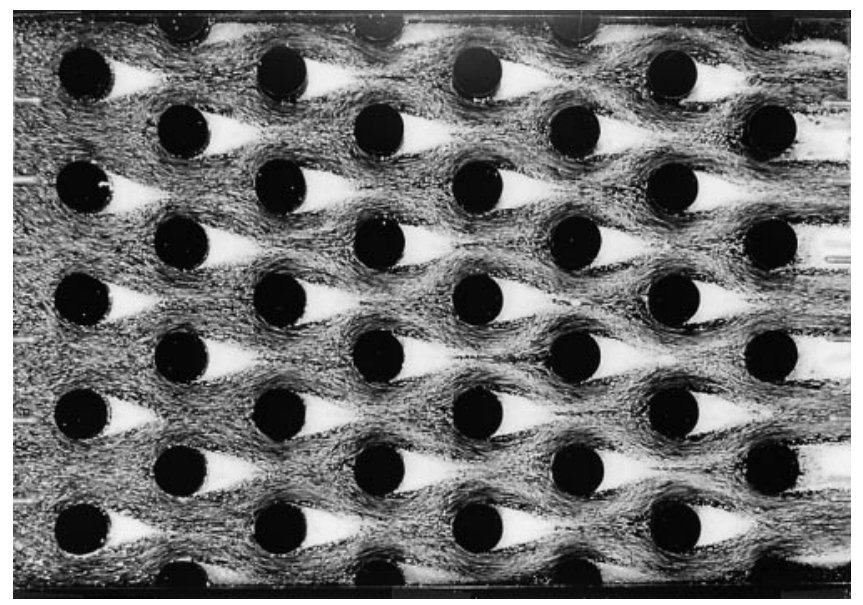

Fig. 8. Flow patterns in type $60-1$ tube bank at $R e, s=610$

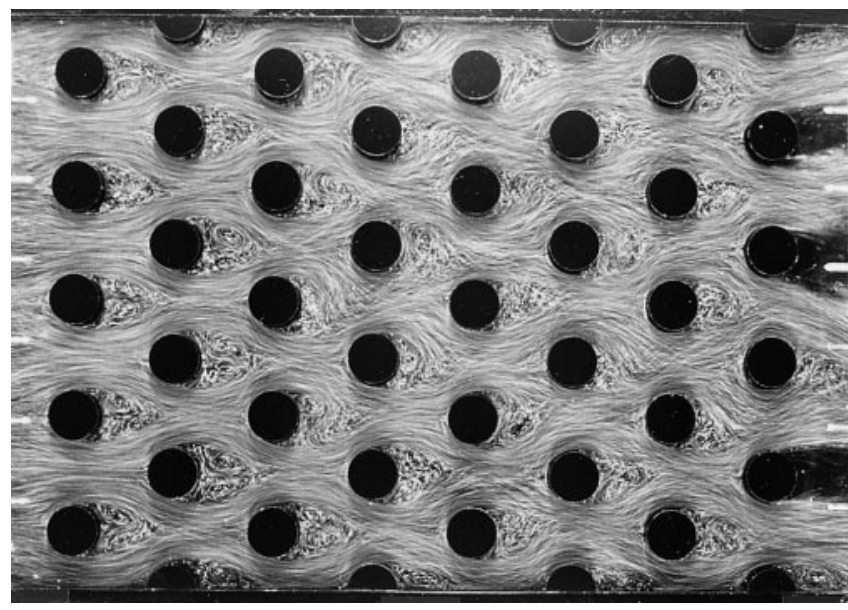

Fig. 9. Flow patterns in type 60-1 tube bank at $R e, s=13140$

of circular curvatures, each with its center located on the extension of the $\mathrm{BC}$ line, a radius curvature, $R$, and tangent to a pair of diagonals (shown by broken line) connecting the midpoints of four sides of the diamond-shaped rectangle $A B C D$. Let the radius ratio of the cylinder to the curvature be $C_{r}=r / R=0.5 \mathrm{D} / R$. Other dimensionless geometric parameters

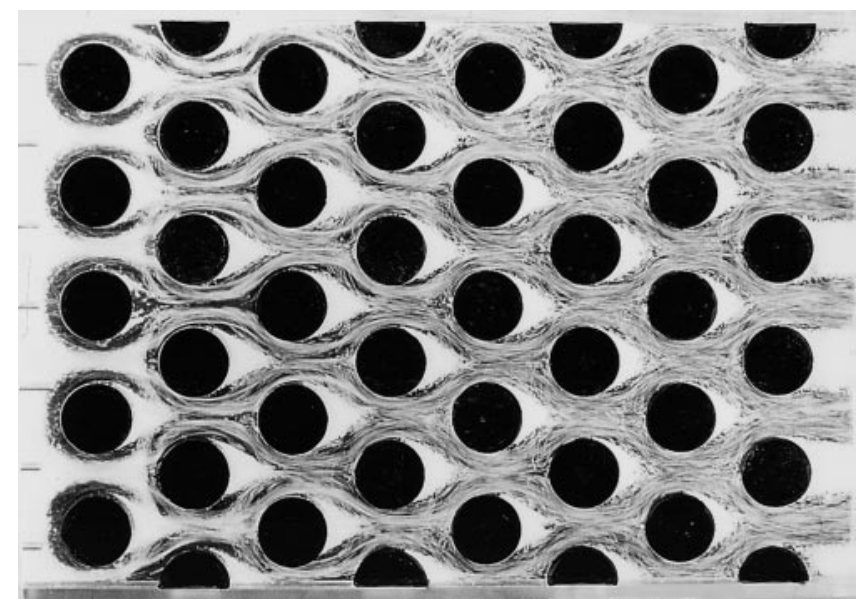

Fig. 10. Flow patterns in type $60-2$ tube bank at $R e, s=700$

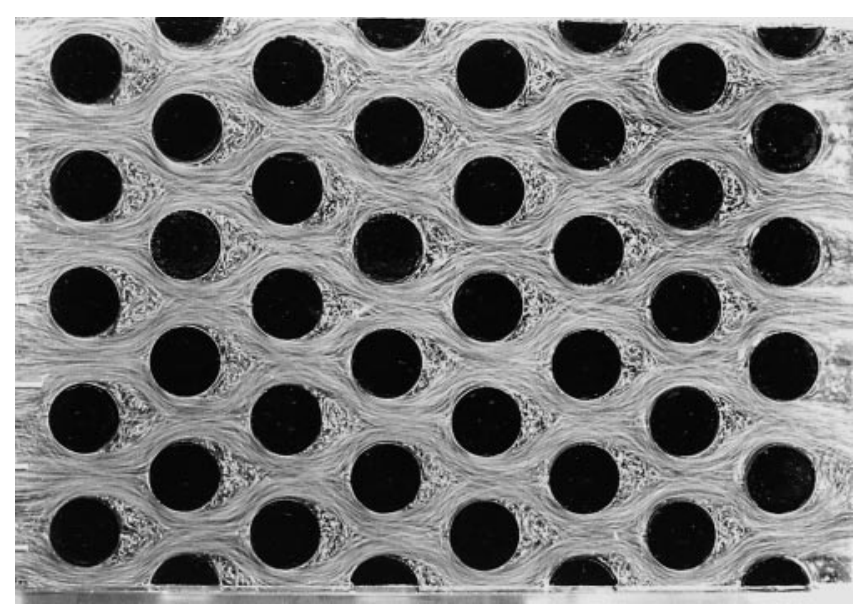

Fig. 11. Flow patterns in type $60-2$ tube bank at $R e, s=14430$

are:

$C_{f}=\left(A_{d}-A_{c}\right) / A_{d}, C_{1}=T_{1} / W, C_{g}=S_{y} / S_{g}, C_{a}=X / Y$

Here,

$A_{d}=$ area of the diamond-shape rectangle $=X Y$

$A_{c}=$ cross sectional area of each cylinder $=\pi D^{2} / 4$

$T_{1}=$ total lateral length of $m$ cylinders in a row

$W=$ width of test section

$S_{y}=$ lateral space between cylinders $=Y-D$

$S_{g}=$ diagonal space between cylinders $=X / \cos (\theta / 2)-D$

$X$ denotes one-half of the longitudinal pitch and $Y$ is the lateral pitch between cylinders. The characteristic length of the cylinder bundle $h_{D}=2 S_{g} d /\left(S_{g}+d\right)$ where $d$ is the depth of liquid flow in the test section such that the Reynolds number can be expressed as $R e=h_{D} U_{\max } / v$. Let $\Delta E$ be the head loss of specific energy $E$ which is the sum of pressure head and velocity head. It is experimentally found that the head loss ratio $\triangle E / L$ depends on fourteen physical quantities. That is, $\Delta E / L=f\left(\rho, \mu, h_{D}, U_{\max }, D, R, A_{d}, A_{c}, W, T_{1}, S_{y}, S_{g}, X, Y\right)$. A dimensional analysis finds $\Delta E / L$ to be the function of six dimensionless parameters, $R_{e}, C_{r}, C_{f}, C_{g}$ and $C_{a}$. Test data are correlated in two different ways: $K i$ versus $R e$ and $K b$ versus $R e$. 


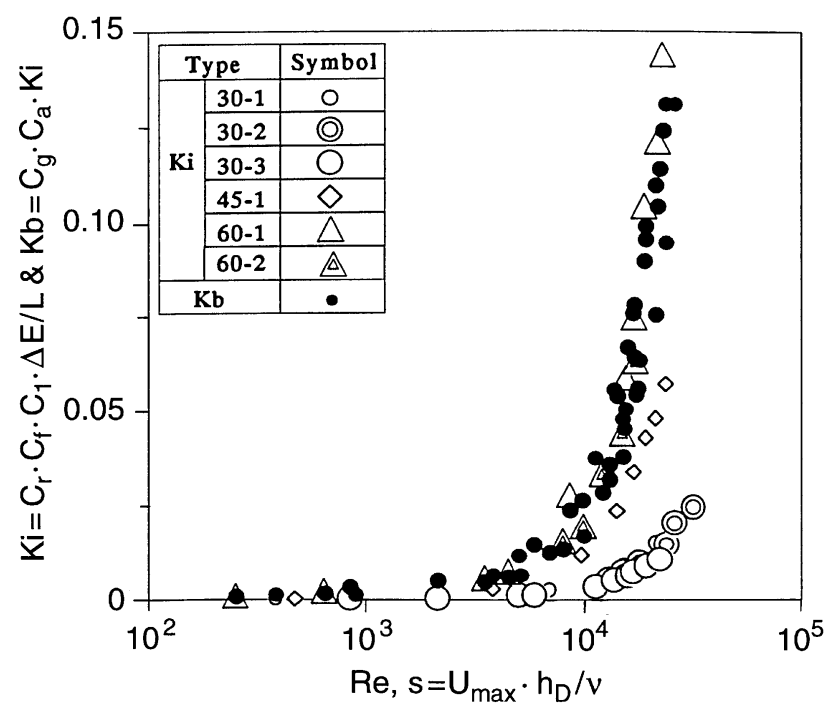

Fig. 12. Friction loss coefficients $K i$ and $K b$ versus $R e, s$

Here, both $K i$ and $K b$ are defined to include $\Delta E / L$ as $K i=C_{r} C_{f} C_{l} \Delta E / L$ and $K b=C_{g} C_{a} K i$,

respectively. Results are graphically presented in Fig. 12. It is seen that $K i$ increases monotonically with $R e$ for all six cylinder bundles of different geometrical arrangement. The $60^{\circ}$ bundles are characterized by the steepest slope in $K i$ with $R e$ and the $30^{\circ}$ ones have the least slope with the $45^{\circ}$ one in between. However, when the data are recorrelated for $K b$ against $R e$, results for all six bundles collapse into a single curve. Such a success in the dimensional analysis followed by experimental correlations suggests that both parameters $K i$ and $K b$ can be used to experimentally determine the friction chracteristics of flow through staggered cylinder bundles. However, with the dimensions specific to cylinder arrangement, $X, Y, S_{g}$ and $S_{y}$ taken into account, $K b$ becomes the parameter free from cylinder arrangement. It should be noted that the test data were taken from stations No. 1 to No. 6 , thus $\Delta E / L$ included the entrance effect into the cylinder bundles.

\section{3}

\section{Power spectrum characteristics}

A power spectrum analysis was performed utilizing the transient component in the $\mathrm{v}$ direction (direction normal to the flow) obtained by $L D V$ measurements at station No. 5 shown in Fig. 1. The timewise variation of auto-correlation is presented in Fig. 13, while representative results for the distribution of the normalized power spectrum are given in Figs. 14 through 16. It is seen in Fig. 13 that auto-correlation is characterized by periodic oscillations. In Fig. 14, there exists an optimum frequency $f_{v}$ where the power spectrum reaches a peak value, indicating that flow in the type 30-1 unit exhibits a distinctive periodic characteristic. The reason for possessing such periodic characteristics is that flow oscillations induced by von Karman vortex streets interact with the intersecting main streams. The occurrence of the optimum frequency is also disclosed in the other units, as seen in Figs. 15 and 16, but is accompanied by high values of power spectra at the neighboring frequencies. They are the power spectra resulting from noise-like transient variations.

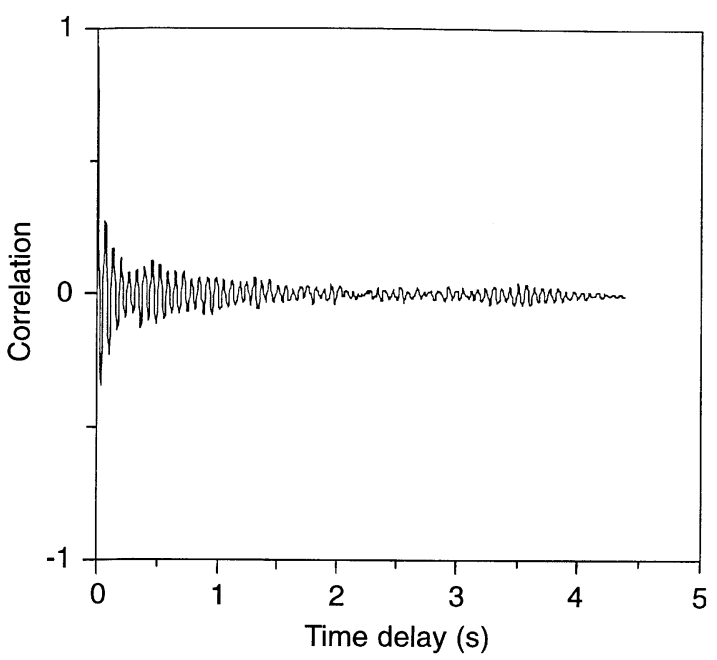

Fig. 13. Auto-correlation in Type 30-1 tube bundle at $R e, s=21060$

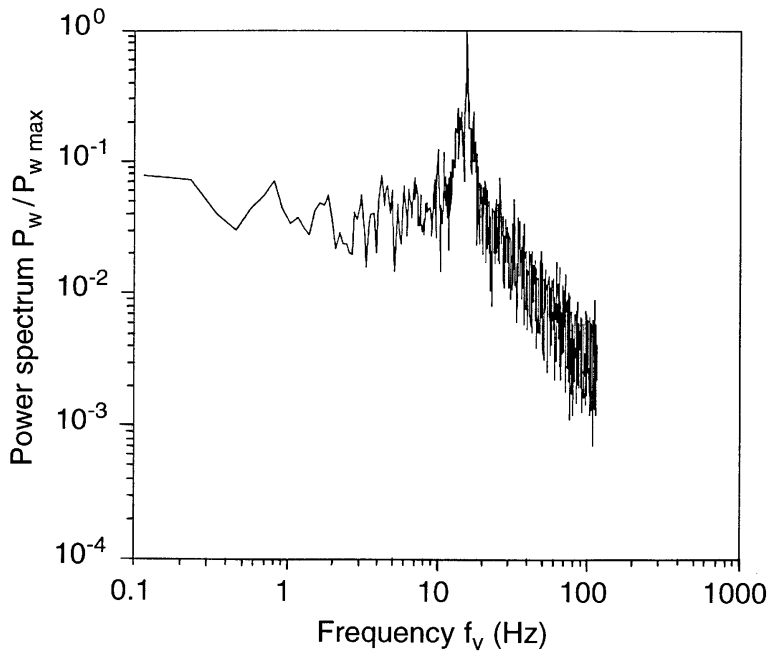

Fig. 14. Power spectrum in Type 30-1 tube bundle at $R e, s=21060$

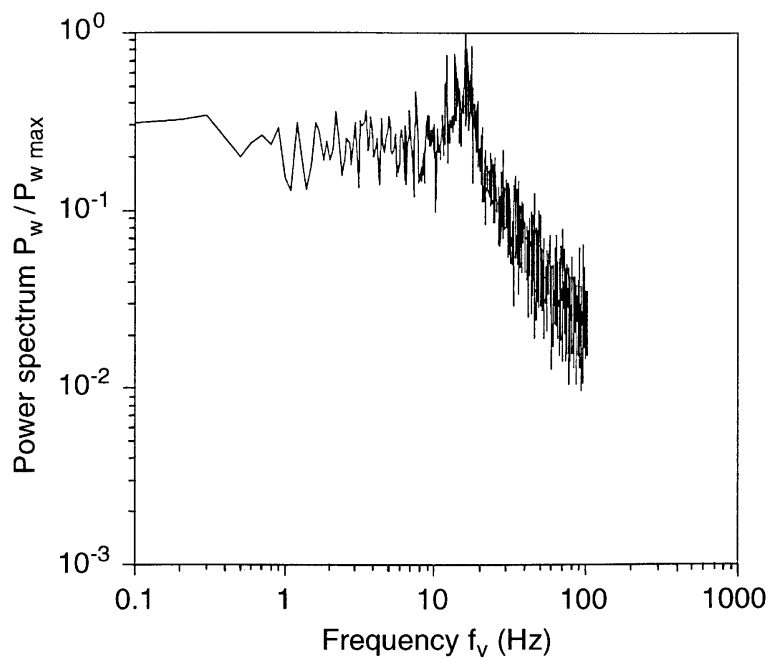

Fig. 15. Power spectrum in Type 30-2 tube bundle at $R e, s=31520$ 


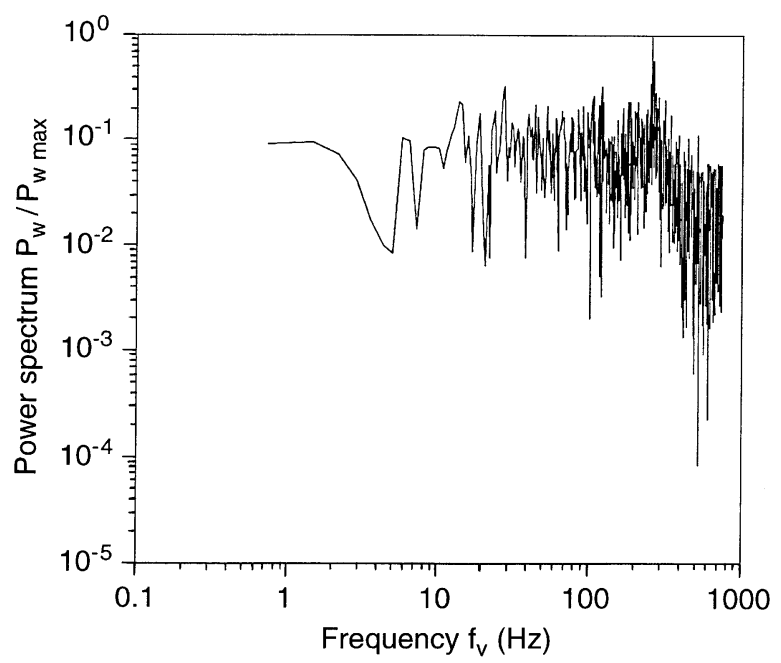

Fig. 16. Power spectrum in Type 60-1 tube bundle at $R e, s=21690$

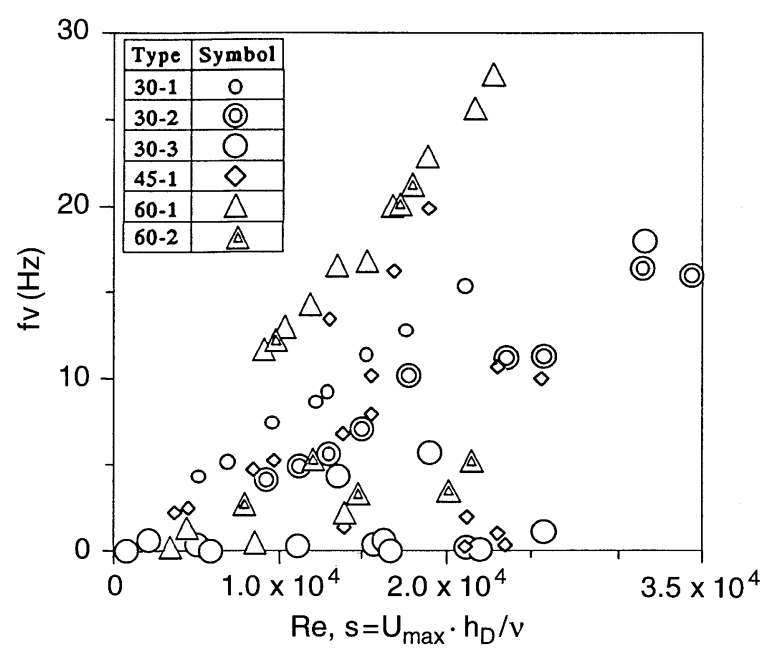

Fig. 17. Optimum frequency $f_{v}$ versus $R e, s$

The variation of the optimum frequency $f_{v}$ with respect to the Reynolds number $R e, s$ is plotted in Fig. 17. It is seen that $f_{v}$ increases $R e, s$ for types 30-1, 30-2 and 60-1. On the other hand, types 30-3 and 45-1 have the values of $f_{v}$ increase $R e, s$ not monotonically but with fluctuation. The reason for $f_{v}$ to fluctuate with $R e, s$ is because the intersecting streams flow around each tube in a manner that confines the wake flow region behind the tube. In other words, the power spectrum has caught simultaneously the oscillation of the von Karman vortex street and that of the intersecting flow. Hence, the effect of noise-like transient components appears in the power spectrum instead of indistinctive periodic characteristics.

Next, the Strouhal number is defined for the optimum frequency $f_{v}$ of only types 30-1, 30-2 and 60-1 in which $f_{v}$ varies monotonically with $R e, s$ as

$S t=f_{v} h_{D} / U_{\max }$

Results are illustrated in Fig. 18. It is seen that each unit has a fairly constant value of $S t$ with respect to $R e, s$.

It is revealed from these results that the power spectrum characteristics for flow through a tube bundle vary depending

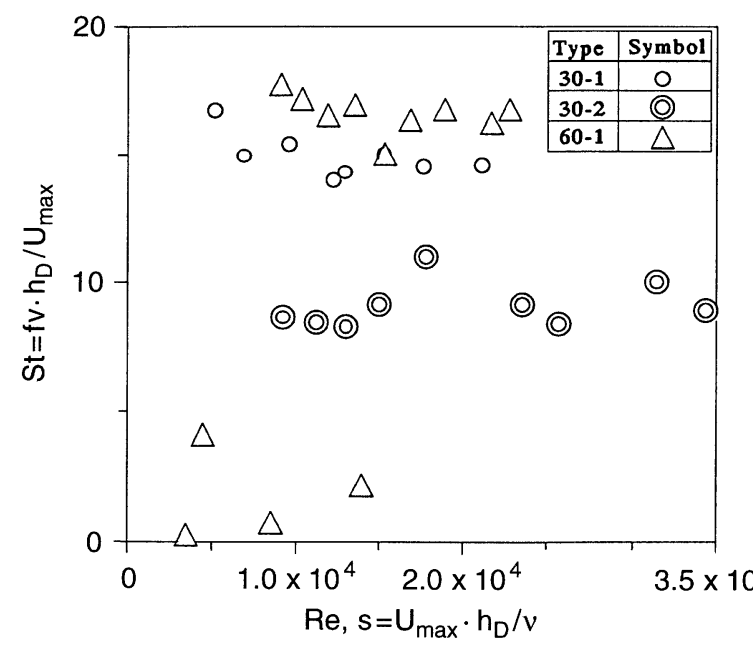

Fig. 18. Strouhal number $S t$ versus $R e, s$

upon the interaction between the vortex behind a tube and the intersecting flow of the main streams induced by the arrangement conditions of the tube bundle. Specifically, the variation of $S t$ remains almost constant with the Reynolds number for those tube bundles with large values of both the longitudinal and lateral pitches ( $X$ and $Y$, respectively). On the contrary, in those densely arranged staggered tube bundles, it is difficult to have a distinct optimum frequency in the power spectrum, due to mixing of the fluctuation of the intersecting flow and that of von Karman vortex street.

\section{4}

\section{Conclusions}

The following conclusions have been derived from the analyses of flow through staggered tube bundles utilizing flow visualization by means of the tracer injection method, piezometry, and $L D V$ flow measurements:

(1) The shape and dimension of a wake vortex formed behind a tube varies depending upon the staggered arrangement angle and the Reynolds number.

(2) Flow conditions around a tube vary with the staggered arrangement angle. For the $30^{\circ}$ angle cases, the oscillation of the von Karman vortex street induces a flip-flop flow. In the case of the $60^{\circ}$ angle, a symmetric flow is formed around a tube, thus confining a wake vortex.

(3) Friction loss characteristics in staggered tube bundles are determined by head loss variation against the Reynolds number for different arrangement angles, with the hydraulic diameter in the diagonal direction of the tube arrangement as the characteristic length.

(4) The power spectrum characteristics of velocity due to different arrangement conditions in the tube bundle can be expressed in terms of the Strouhal number which is uniquely related to the Reynolds number.

\section{References}

Chen SS (1987) Flow-induced vibration of circular cylindrical structures, pp 303-344. Hemisphere Publishing Corp, New York

Moretti PM (1993) Flow-induced vibrations in arrys of cylinders. Ann Rev Fluid Mech 25: 99-114 
Nakai T; Ayukawa K; Inoue T (1996) Flow through dense tube bundles, Part 1: pressure distribution on tube surface and velocity variation inside tube bundles. Trans Japan Soc Mech Eng Series B. 62: 65-71

Rabbo AA; Weaver DS (1986) A flow visualization study of tube array. J Sound Vibration 106: 241-256

Umeda S; Yang Wen-Jei (1995) Flow visualization inside vertical intersecting ducts - flow resistance and characteristics. Visualization Soc Japan 15: 41-46

Umeda S; Yang Wen-Jei (1996a) Flow characteristics in flow networks. J Visualization Soc Japan 16: 28-36

Umeda S; Yang Wen-Jei (1996b) Flow visualization in intersecting ducts with different converging-diverging angles. J Visualization Soc Japan 16: 19-27

Weaver DS; Fitzpatrick JA (1988) A review of cross-flow induced vibrations in heat exchanger tube arrays. J Fluids Structures 2: 73-93

Werle H (1972) Sur l'ecoulement autour d'um faisceau tubulaire. Revue Francaise de Mechanique 41: 7-19 